\title{
95 Muskelkater
}

C) Springer-Verlag GmbH Deutschland, ein Teil von Springer Nature 2018

D. Mathias, Fit und gesund von 1 bis Hundert

https://doi.org/10.1007/978-3-662-56307-6_95

Nach ungewohnten oder besonders intensiven sportlichen Übungen stellen sich häufig Schmerzen in der beanspruchten Muskulatur ein. Die betroffenen Muskeln werden als kraftlos, hart und druckempfindlich wahrgenommen. Als Ursache für den Muskelkater (ehemals Muskelkatarrh) gelten Einrisse bei einem Teil der Muskelfasern speziell im Bereich der Z-Scheiben. Durch die leicht geschädigten Muskelfasern dringt langsam Wasser ein, die Fasern schwellen an und diese Dehnungen lösen Schmerz aus. Zusätzlich führen die Dehnungen zu Gefäßeinengungen mit Verschlechterungen der Durchblutung und weiterer Schmerzsteigerung. So dauert es 1-3 Tage, bis das Schmerzempfinden seinen Höhepunkt erreicht und dann langsam wieder abklingt. Als Folge des Abbaus der zerstörten Fasern kommt es zu ste- rilen Entzündungen. Die jetzt einsetzenden Reparaturmechanismen hinterlassen einen etwas stärkeren Muskel. Nach etwa drei erhöhten Beanspruchungen hat sich der betroffene Muskel so an die neuen Anforderungen angepasst, dass der »Katarrh" ausbleibt.

Weil die Muskelfasern ihre größten Kräfte bei exzentrischen Kontraktionen entfalten ( $\triangleright$ Kap. 85), sind es auch diese Beanspruchungen, die am häufigsten Muskelkater auslösen. Der Muskelkater hinterlässt keine bleibenden Schäden und ist durch gleiche Bewegungen für mehrere Wochen nicht erneut auslösbar. Durch vorsichtiges Dehnen und leichte dynamische Bewegungen lassen sich seine Symptome etwas abmildern. Auch Saunagänge oder warme Bäder können etwas helfen. Ein Wundermittel, das die Dauer des Muskelkaters verkürzt, existiert nicht. Nach 3-4 Tagen ist er von allein verschwunden.

- Abb. 95.1 Nach den Anstrengungen duscht sich ein Mann mit kaltem Wasser, wie Hippokrates dies empfohlen hat (etwa 300 v. Chr). (Mit freundlicher Genehmigung Andreas Verlag, Salzburg) 